\title{
Mortality following operations for lower extremity peripheral arterial disease
}

\author{
This article was published in the following Dove Press journal: \\ Vascular Health and Risk Management \\ 30 April 2010 \\ Number of times this article has been viewed
}

\author{
Tracie C Collins ${ }^{1,2}$ \\ David Nelson ${ }^{3}$ \\ Jasjit S Ahluwalia ${ }^{1,2}$ \\ 'Department of Medicine, Division of \\ General Internal Medicine, University \\ of Minnesota Minneapolis, MN, USA; \\ ${ }^{2}$ Center for Health Equity, University \\ of Minnesota Medical School, \\ Minneapolis, MN, USA; ${ }^{3}$ Minneapolis \\ VA Medical Center, Center for \\ Chronic Disease Outcomes Research, \\ University of Minnesota, Minneapolis, \\ MN, USA
}

Background: We sought to identify risk factors associated with mortality following surgery for peripheral arterial disease (PAD).

Methods: We evaluated the association between levels of control of atherosclerotic risk factors and time to mortality following either lower extremity bypass surgery or lower extremity amputation using Cox proportional hazards regression.

Results: Among 796 patients with PAD (defined by an ankle-brachial index $[\mathrm{ABI}]<0.9$ ), 230 (28.9\%) underwent an operation for PAD (136, lower-extremity bypasses; 111, lower-extremity amputations). Participants were followed for up to six years after their diagnosis of PAD. A total of 107 (46.5\% of the 230) died during the period of follow-up. Factors associated with mortality following lower extremity bypass surgery included age 70 years and older hazard ratio [HR] 1.88; 95\% confidence interval [CI]: 1.01-3.51) and of African American race (HR 1.94; 95\% CI: 1.04-3.62). Renal insufficiency was significantly associated with mortality following lower extremity amputation (HR 2.19; 95\% CI: 1.16-4.13).

Conclusion: Our data provide information on preoperative risk variables to consider when assessing long-term mortality in persons with PAD who are undergoing surgery for PAD.

Keywords: risk factors, mortality, bypass surgery, ankle-brachial index

\section{Introduction}

Peripheral arterial disease (PAD) affects 8-12 million Americans. ${ }^{1,2}$ The primary goal in managing PAD is to reduce both cardiovascular events, such as myocardial infarction or stroke, and limb events, such as progressive ischemia that requires lower-extremity surgical bypass or amputation. ${ }^{1,3}$ Leg amputation is associated with ongoing impairment of functional status and quality-of-life. ${ }^{4}$ Although less devastating than lower extremity amputation, lower-extremity bypass surgery is associated with 30-day mortality from $2 \%$ to $8 \%$ and five-year graft failure rates from $50 \%$ to $90 \%$ with higher rates of failure for persons with critical limb ischemia versus claudication. ${ }^{5}$ In addition, PAD patients who undergo lower extremity bypass surgery have a $20 \%$ risk of amputation within 5 years. ${ }^{3,6}$

Aside from surgical therapy, risk factor modification is an important treatment modality for PAD. Risk factors for PAD include smoking, diabetes mellitus, hypertension, and dyslipidemia. ${ }^{7-9}$ Treatment of these risk factors should, optimally, reduce both systemic outcomes (such as myocardial infarction and stroke) and surgical limb events. For patients who require limb surgery, the risk for postoperative survival should be optimized. The focus of this work was to determine risk factors for mortality following lower extremity revascularization or amputation for PAD.
Correspondence:Tracie C Collins Department of Medicine, University of Minnesota, 420 Delaware St., S.E. MMC 45I Minneapolis, MN 55455 USA

$\mathrm{Tel}+\mathrm{l} 6126250415$

Fax +I 6126266782

Email tcc@umn.edu 


\section{Methods}

\section{Study population}

Consecutive patients evaluated at the Michael E DeBakey Veterans Affairs Medical Center (MEDVAMC) noninvasive vascular laboratory from 1995 to 1998 with a diagnosis of PAD based on an ankle-brachial index (ABI) of $<0.90$ were included in this study. Patients who had undergone a prior lower extremity vascular surgical revascularization procedure or a major amputation were excluded. The Baylor College of Medicine Institutional Review Board approved this study.

Detailed information for the methods of this retrospective cohort study has been previously published. ${ }^{10}$ We obtained information on first limb event (bypass surgery or amputation) and mortality through the end of the observation period (December 31, 2001). Our retrospective cohort included patient record abstractions starting as early as January 1, 1992 and ending with medical records dated at the time of the surgical limb event, death, or December 31, 2001, whichever occurred first.

\section{Data source}

We used medical records from the Veterans Integrated Systems Technology Architecture (VISTA) to identify our cohort and identified outcomes.

\section{Primary outcome}

The primary outcome was all-cause mortality following lower extremity bypass surgery or lower extremity amputation as individual surgical events. Lower extremity bypass surgeries included aortoiliac or aortobifemoral, femoropopliteal bypass, above or below the knee, and femorotibial vascular bypass. Lower extremity amputations included below-the-knee and above-the-knee major procedures (transmetatarsal or toe amputations were not included). Patients who had undergone lower extremity bypass surgery followed by amputation of the same or contralateral extremity were considered in both analyses. This study did not evaluate mortality following endovascular procedures as these procedures were not performed in large numbers until after 2001.

\section{Independent variables}

In addition to standard patient demographic measures, potential correlates of mortality included levels of actual risk factor control (separately for smoking cessation, glycemic control, blood pressure control, and lipid control). We also tracked cohort members' exposure to pharmacological therapy for each of the four risk factors as well as antiplatelet therapy.

\section{Data collection}

Trained nurse chart abstractors blinded to the study purpose completed the chart reviews using standardized data extraction forms developed by study investigators. The nurses captured information on the patient's age, dates of clinic visits, reason for clinic visits, symptoms, physical examination findings (eg, vital signs), diagnoses, and modifiable risk factors. Mortality and additional study data were abstracted from the facility electronic medical record and from the national Veterans Affairs administrative files including the Beneficiary Identification and Records Locator Subsystem (BIRLS) Mortality File and the patient treatment file (PTF).

\section{Risk factor control}

We determined effective control of a risk factor based on documentation from charted vital signs or extracted laboratory data. We defined control of each risk factor according to national guidelines, including those of the National Cholesterol Education Panel; Joint National Committee on Detection, Evaluation, and Management of Hypertension; American Diabetes Association; and American Cancer Society. ${ }^{11-15}$

\section{Statistical analysis}

We implemented a bootstrap model selection procedure, as discussed by Austin and Tu, ${ }^{16}$ to develop predictive models for survival after bypass and for survival after amputation. To develop each model we used 250 replications of the bootstrapping process described forthwith. Within each replication we created a simple bootstrapped version of the sample and implemented a stepwise model selection procedure to construct a Cox proportional hazards survival model using the covariates, described in detail below, as potential predictors. The model selection procedure used $P$-value based selection and retention criteria of 0.20 and 0.25 , respectively, for the significance tests of individual predictors. Such liberal criteria are often chosen to reduce biased estimation of regression parameters with model selection procedures, as discussed by Greenland, ${ }^{17}$ Mickey and Greenland. ${ }^{18}$ Those predictors selected in more than one-half of these bootstrapped models were included in the subsequent Cox proportional hazards regression model fit to the original sample.

The covariates considered as potential predictors included race, index ABI severity, history of coronary artery disease (CAD), most recent serum creatinine prior to surgery, use of ace inhibitors, beta-blockers, calcium channel blockers, and PAD medications within three months of surgery. The model development process also considered smoking, hypertension, cholesterol, and diabetes risk factors together with measures 
of the control of these risk factors and use of medications to treat these risk factors within the three months prior to surgery as potential predictors. These latter medications included diuretics and other antihypertensive medications, diabetes medications, lipid lowering medications, and smoking cessation medications.

Measures of risk factor control were constructed as the proportion of risk assessments (ie, blood pressure, lipid panel, blood glucose) prior to surgery indicating the risk factor was controlled. For a large proportion of individuals, up to $33 \%$ for some risk factors, there was no risk assessment performed or documented in their records. For these individuals, we imputed a proportion of control using results of an overdispersed logistic regression model, fit using the data for those with assessments, for the number of assessments indicating control over the number of assessments using the above covariates as predictors.

\section{Results}

We identified 796 veterans with PAD among whom 230 patients underwent lower extremity bypass surgery or lower extremity amputation. The following characteristics describe those veterans who were treated with lower extremity bypass surgery or underwent a lower extremity amputation (Table 1). The mean age of veterans who experienced an adverse limb event was $64.1 \pm 10.1$ years; 99\% were men; 83 (36.1\%) were African American; 15 (6.5\%) were Latino; and 132 (57.4\%) were white or of unknown race. Individuals were classified according to the tertiles of index PAD severity: mild $(0.70-0.89)$, moderate $(0.41-0.69)$, and severe $(<0.40)$.

Table I Baseline characteristics of the cohort

\begin{tabular}{|c|c|c|c|}
\hline Variable & Total cohort $(N=230)$ & $\begin{array}{l}\text { Patients undergoing } \\
\text { bypass }(N=136)\end{array}$ & $\begin{array}{l}\text { Patients undergoing } \\
\text { amputation }(\mathrm{N}=\mathrm{I} I \mathrm{I})\end{array}$ \\
\hline \multicolumn{4}{|l|}{ Age } \\
\hline Mean age (SD) & $64.1(10.1)$ & $62.5(9.9)$ & $66.6(9.9)$ \\
\hline Age $<60$ years & $66(28.7 \%)$ & 44 (32.4\%) & $24(21.6 \%)$ \\
\hline Age $60-70$ years & 94 (40.9\%) & $60(44.1 \%)$ & $42(37.8 \%)$ \\
\hline Age 70 years and older & 70 (30.4\%) & $32(23.5 \%)$ & 45 (40.5\%) \\
\hline \multicolumn{4}{|l|}{ Sex } \\
\hline Male & $228(99.1 \%)$ & I 34 (98.5\%) & III (I00\%) \\
\hline \multicolumn{4}{|l|}{ Race/Ethnicity } \\
\hline African American & $83(36.1 \%)$ & $4 \mathrm{I}(30.2 \%)$ & 49 (44.1\%) \\
\hline Latino & 15 (6.5\%) & $4(2.9 \%)$ & $12(10.8 \%)$ \\
\hline White/Other/Unknown & $132(57.4 \%)$ & 91 (66.9\%) & $50(45.1 \%)$ \\
\hline \multicolumn{4}{|l|}{$A B I$ severity } \\
\hline Mean ABI (SD) & $0.47(0.16)$ & $0.47(0.14)$ & $0.47(0.18)$ \\
\hline Critical $\leq 0.40$ & $66(28.7 \%)$ & 37 (27.2\%) & 38 (34.2\%) \\
\hline Moderate $0.4 \mathrm{I}$ to 0.69 & 147 (63.9\%) & $92(67.7 \%)$ & $63(56.8 \%)$ \\
\hline Mild 0.70-0.89 & 17 (7.4\%) & $7(5.1 \%)$ & $10(9.0 \%)$ \\
\hline \multicolumn{4}{|l|}{ Creatinine levels } \\
\hline None & $33(14.4 \%)$ & $21(15.4 \%)$ & $14(12.6 \%)$ \\
\hline$<1.0$ & 70 (30.4\%) & $43(31.6 \%)$ & $36(32.4 \%)$ \\
\hline $1.0-1.5$ & $88(38.3 \%)$ & $58(42.7 \%)$ & 35 (31.5\%) \\
\hline I.6 or higher & 39 (I7.0\%) & $14(10.3 \%)$ & $26(23.4 \%)$ \\
\hline \multicolumn{4}{|c|}{ Atherosclerotic risk factors } \\
\hline Diabetes mellitus & 148 (64.4\%) & 70 (51.5\%) & $88(79.3 \%)$ \\
\hline Hypertension & I8I (78.7\%) & $102(75.0 \%)$ & $90(81.1 \%)$ \\
\hline Hyperlipidemia & $|4|(6 \mid .3 \%)$ & $90(66.2 \%)$ & $58(52.3 \%)$ \\
\hline \multicolumn{4}{|l|}{ Recent smoking status } \\
\hline Current smoking & $8 \mathrm{I}(35.2 \%)$ & 59 (43.4\%) & $29(26.1 \%)$ \\
\hline Nonsmoking & $14(6.1 \%)$ & $6(4.4 \%)$ & $8(7.2 \%)$ \\
\hline Not documented & $135(58.7 \%)$ & 71 (52.2\%) & 74 (66.7\%) \\
\hline
\end{tabular}


Table I (Continued)

\begin{tabular}{llll}
\hline Variable & Total cohort $(\mathbf{N}=\mathbf{2 3 0})$ & $\begin{array}{l}\text { Patients undergoing } \\
\text { bypass }(\mathbf{N}=\mathbf{1 3 6})\end{array}$ & $\begin{array}{l}\text { Patients undergoing } \\
\text { amputation }(\mathbf{N}=\mathbf{I} \text { I I })\end{array}$ \\
\hline Medication use & $38(16.5 \%)$ & $20(14.7 \%)$ & $19(17.1 \%)$ \\
ACE inhibitor & $20(8.7 \%)$ & $10(7.4 \%)$ & $10(9.0 \%)$ \\
Beta blocker & $44(19.1 \%)$ & $28(20.6 \%)$ & $17(15.3 \%)$ \\
Calcium channel blocker & $45(19.6 \%)$ & $18(13.2 \%)$ & $29(26.1 \%)$ \\
Diuretic & $98(42.6 \%)$ & $50(36.8 \%)$ & $50(45.0 \%)$ \\
Antihypertensive medication & $20(8.7 \%)$ & $15(11.0 \%)$ & $5(4.5 \%)$ \\
Lipid-lowering medication & $59(25.6 \%)$ & $18(13.2 \%)$ & $42(37.8 \%)$ \\
Diabetes medication & $2(0.9 \%)$ & $2(0.9 \%)$ & $0(0.0 \%)$ \\
Smoking cessation medication & $69(30.0 \%)$ & $35(25.7 \%)$ & $37(33.3 \%)$ \\
PAD medication & & & \\
\hline
\end{tabular}

Notes: *Patients may have undergone more than one type of limb surgery.

Abbreviations: $A B I$, ankle-brachial index; $A C E$, angiotensin converting enzyme; $P A D$, peripheral artery disease.

As defined by the index ABI level, the prevalence of critical PAD was $28.7 \%$, moderately severe disease was $63.9 \%$, and mildly severe disease was $7.4 \%$. As captured any time during the observation period, 148 (64.4\%) had diabetes mellitus, $141(61.3 \%)$ had elevated low-density lipoprotein (LDL), and $181(78.7 \%)$ had hypertension.

Among those undergoing lower extremity bypass surgeries, African Americans comprised 30.2\%, Latinos comprised 2.9\%, and non-Hispanic whites comprised $66.9 \%$ of this cohort. In contrast, African Americans and Latinos comprised $44.1 \%$ and $10.8 \%$, respectively, of the cohort undergoing lower extremity amputations while non-Hispanic whites comprised $45.1 \%$.

The risk factors identified for incorporation in the model for mortality following lower extremity bypass surgery were African American race hazard ratio [HR] 1.94; 95\% confidence interval [CI] 1.04-3.62), age 70 years or older (HR $1.88,95 \%$ confidence interval [CI]: 1.01-3.51), and index ABI (HR 0.12; 95\% CI: 0.01-1.09) (Table 2). Figures 1-3 present model-based estimated survival curves for survival after bypass by age, index ABI, and race/ethnicity. In constructing the survival curve estimates for each figure, the other predictors were fixed at the most prevalent or median level.

Risk factors identifed for inclusion in the model for mortality following lower extremity amputation included index ABI, smoking status, history of CAD, latest serum creatinine, and days between index and amputation. While the standard regression test results for the significance of $\mathrm{ABI}$ and smoking are marginally significant at best, the model-estimated coefficients for these predictors are generally consistent with expectations. Interestingly, the model suggests that nonassessment of smoking status is associated with increased mortality. As illustrated in Table 2, history of CAD is associated with increased mortality (HR 1.79; 95\% CI: 1.05-3.05). Similarly, elevated serum creatinine is associated with increased mortality with approximate doubling of the hazard, relative to a serum creatinine less than 1, between 1 and 1.5 (HR 2.12; 95\% CI: 1.11-4.03), and between 1.5 and 2.0 (HR 2.50, 95\% CI: 1.05-5.93). An

Table 2 Risk factors for mortality following each type of surgery

\begin{tabular}{|c|c|c|c|}
\hline Variable & $P$ value & Hazard ratio & $\begin{array}{l}95 \% \text { confidence } \\
\text { limits }\end{array}$ \\
\hline \multicolumn{4}{|c|}{ Lower extremity bypass surgery } \\
\hline Index $A B \mid$ & 0.059 & 0.12 & $0.01,1.09$ \\
\hline Age 70 years and older & 0.048 & 1.88 & I.0I, $3.5 \mathrm{I}$ \\
\hline $\begin{array}{l}\text { African American race/ } \\
\text { ethnicity }\end{array}$ & 0.038 & 1.94 & $1.04,3.62$ \\
\hline \multicolumn{4}{|c|}{ Lower extremity amputation } \\
\hline Index ABI & 0.089 & 1.46 & $0.90,2.35$ \\
\hline $\begin{array}{l}\text { Days between index and } \\
\text { amputation }\end{array}$ & 0.066 & 1.001 & $1.000,1.002$ \\
\hline \multicolumn{4}{|l|}{ Recent smoking status } \\
\hline Current smoker & 0.122 & 0.58 & $0.29,1.16$ \\
\hline Nonsmoker & 0.076 & 0.37 & $0.12,1.11$ \\
\hline History of CAD & 0.034 & 1.79 & $1.05,3.05$ \\
\hline \multicolumn{4}{|l|}{ Serum creatinine* } \\
\hline 1.0 to 1.5 & 0.022 & 2.19 & $1.16,4.13$ \\
\hline 1.5 to 2.0 & 0.038 & 3.84 & $1.94,7.62$ \\
\hline 2.0 or greater & $<0.001$ & 4.51 & $2.00,10.17$ \\
\hline Unmeasured & 0.068 & 2.12 & $0.95,4.77$ \\
\hline
\end{tabular}

Notes: ${ }^{\#}$-value for overall test for smoking status $=0.089$, no assessment of smoking status was used as reference.

$* P$-value for overall test for serum creatinine effects $=0.008$, unknown status used as reference. 


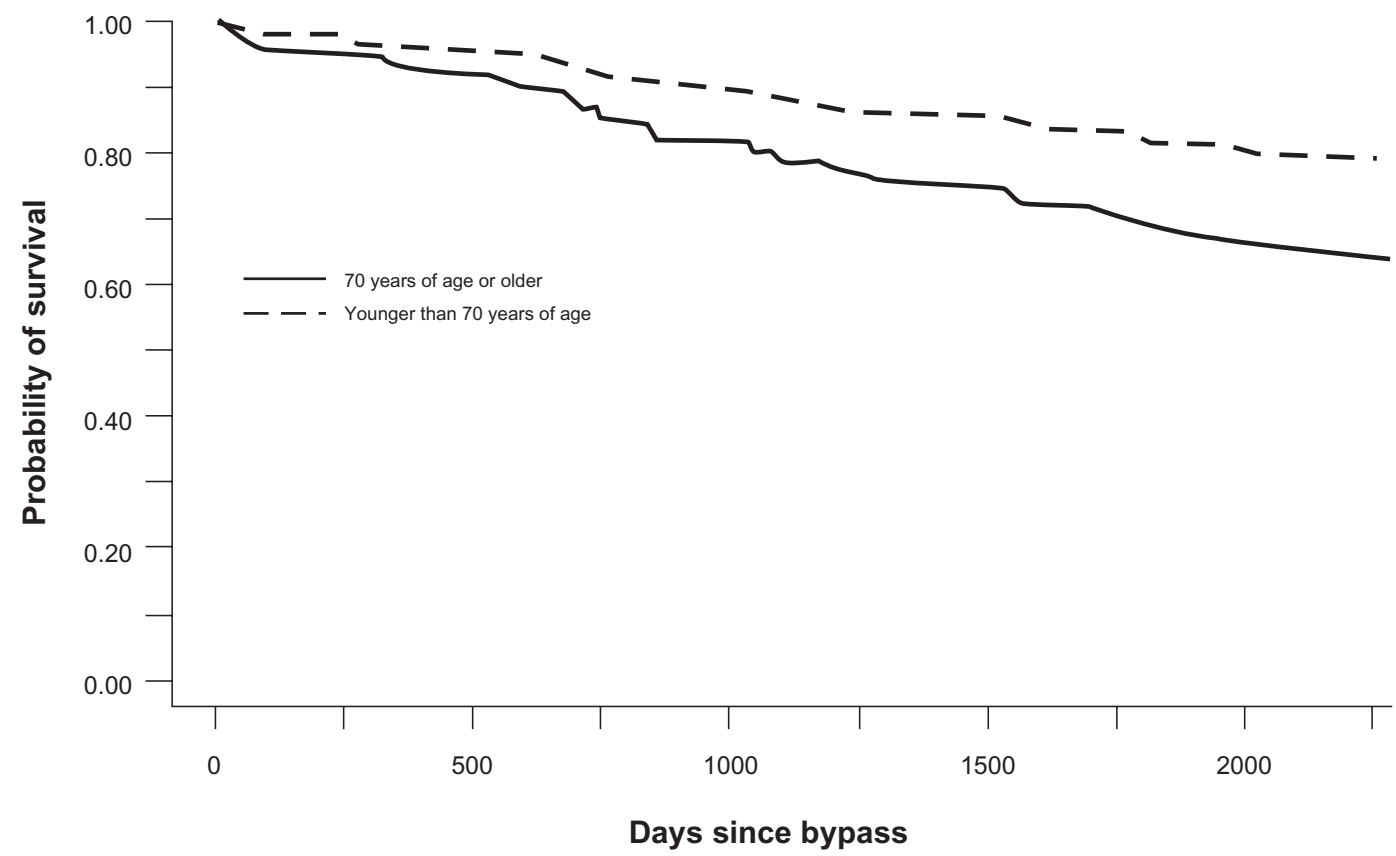

Figure I Model-based estimated survival curves for survival after bypass by age.

even larger increase in the hazard was observed for serum creatinines greater than 2.0 (HR 4.51; 95\% CI: 2.00-10.17). Figures 4-7 present model-based estimated survival curves for survival after amputation bypass using smoking status, index ABI, history of CAD, and serum creatinine. Here again, in constructing the survival curve estimates for each figure, the other predictors were fixed at the most prevalent or median level.

\section{Discussion}

We identified age, disease severity, African American race, renal insufficiency at all levels (including a range of

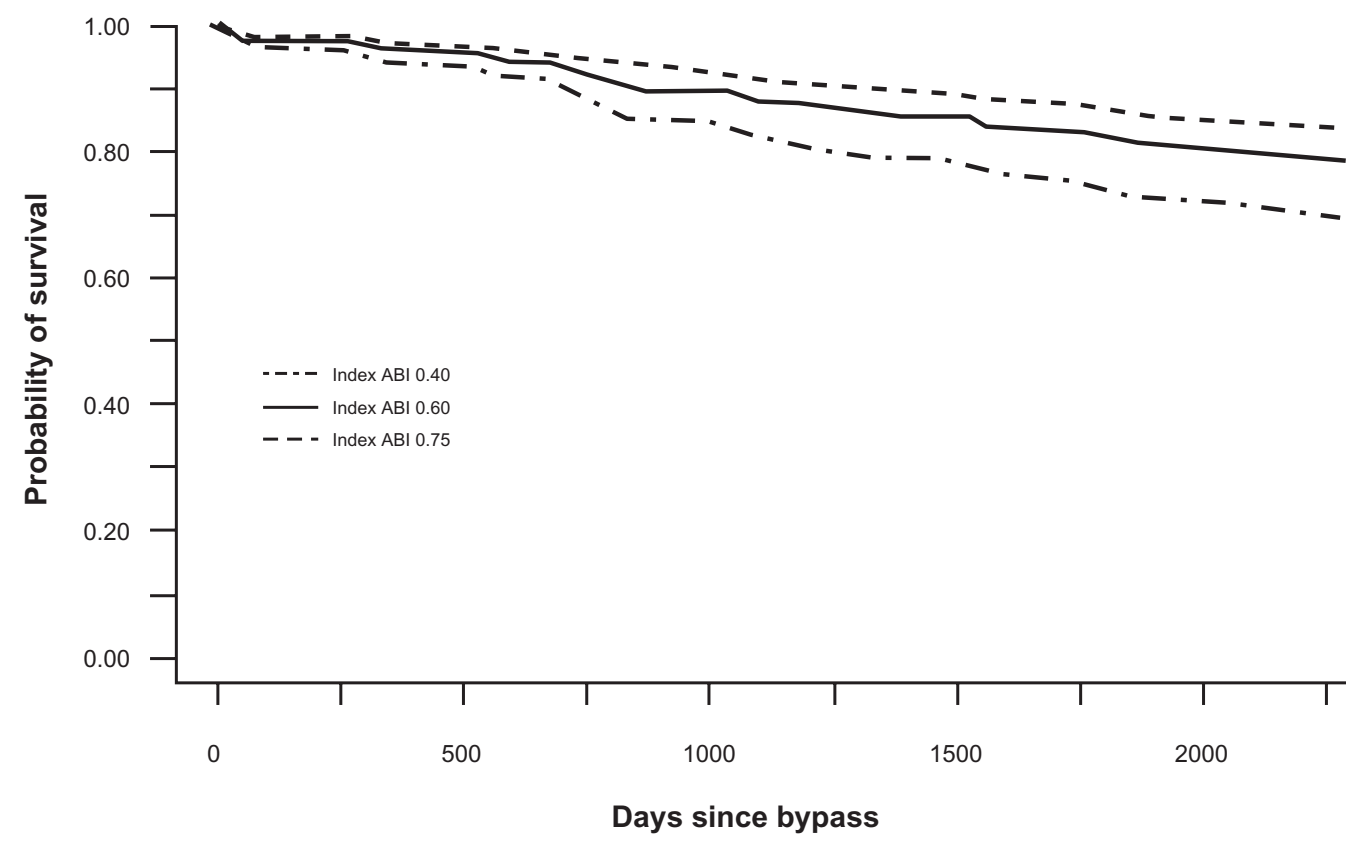

Figure 2 Model-based estimated survival curves for survival after bypass by index $A B I$. Abbreviation: $A B I$, ankle-brachial index. 


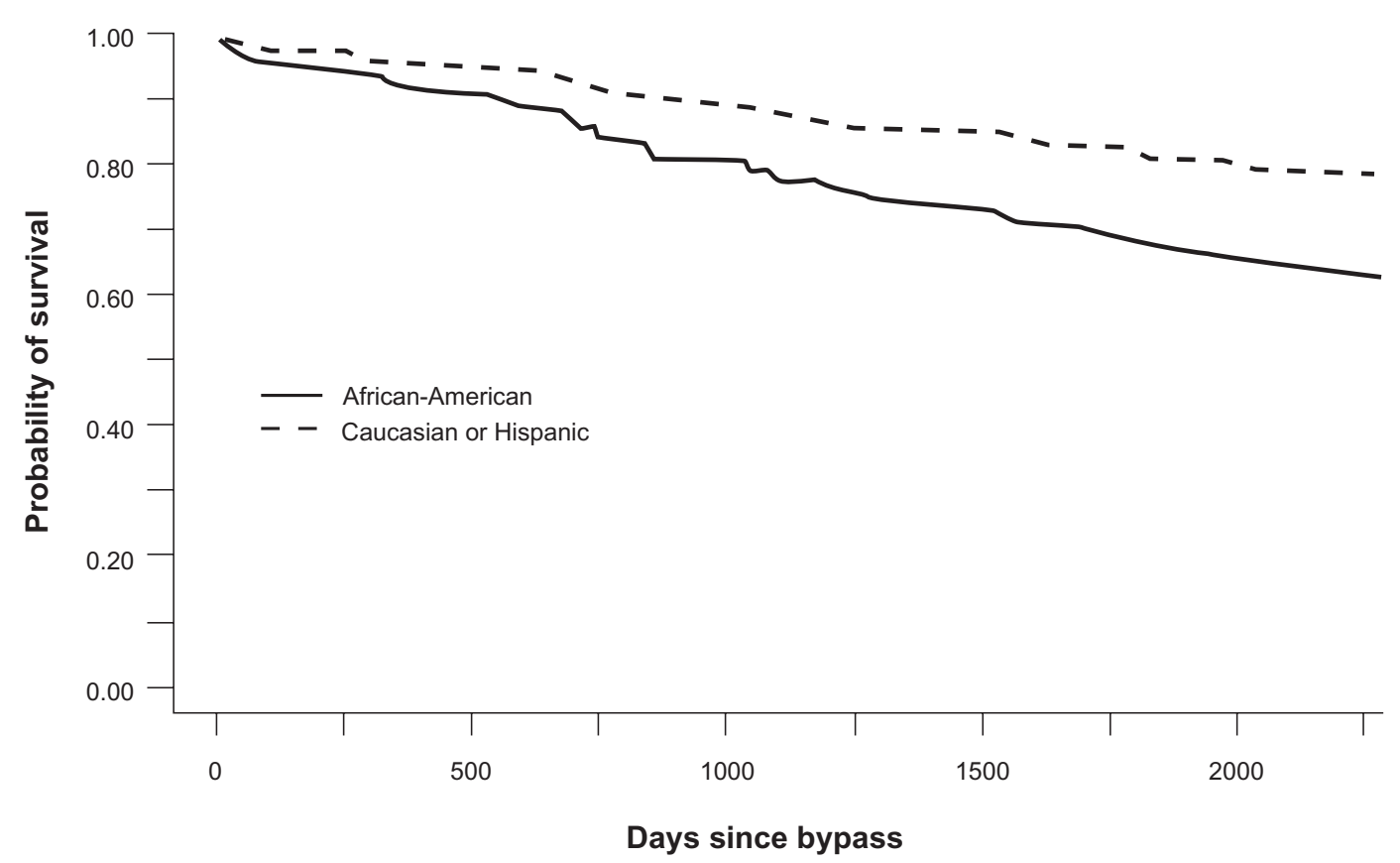

Figure 3 Model-based estimated survival curves for survival after bypass by race/ethnicity.

normal serum creatinine to mild elevation), nonassessment of smoking status, and a history of coronary artery disease as risk factors associated with mortality following lower extremity bypass surgery and lower extremity amputation.

Advanced age is a known risk factor for PAD and other cardiovascular diseases. ${ }^{19,20}$ In addition, persons who are aged at least 65 years or older have an increased risk for mortality following both vascular and nonvascular surgery. We found that veterans aged 70 years and older were at an increased risk for mortality following lower extremity bypass surgery. As the proportion of US adults aged 70 years and older increases, efforts to optimize risk factor status prior to

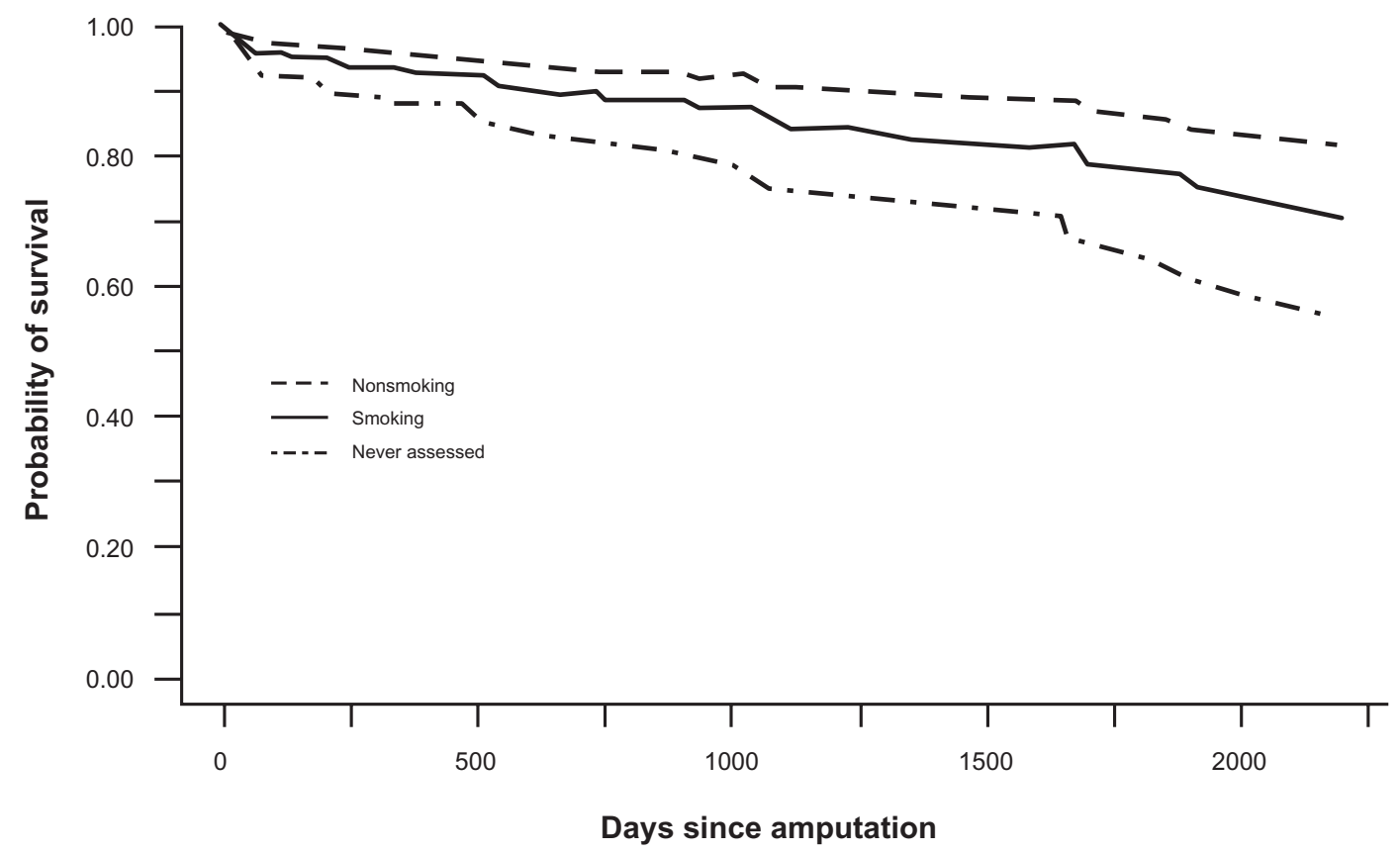

Figure 4 Model-based estimated survival curves for survival after amputation by smoking status. 


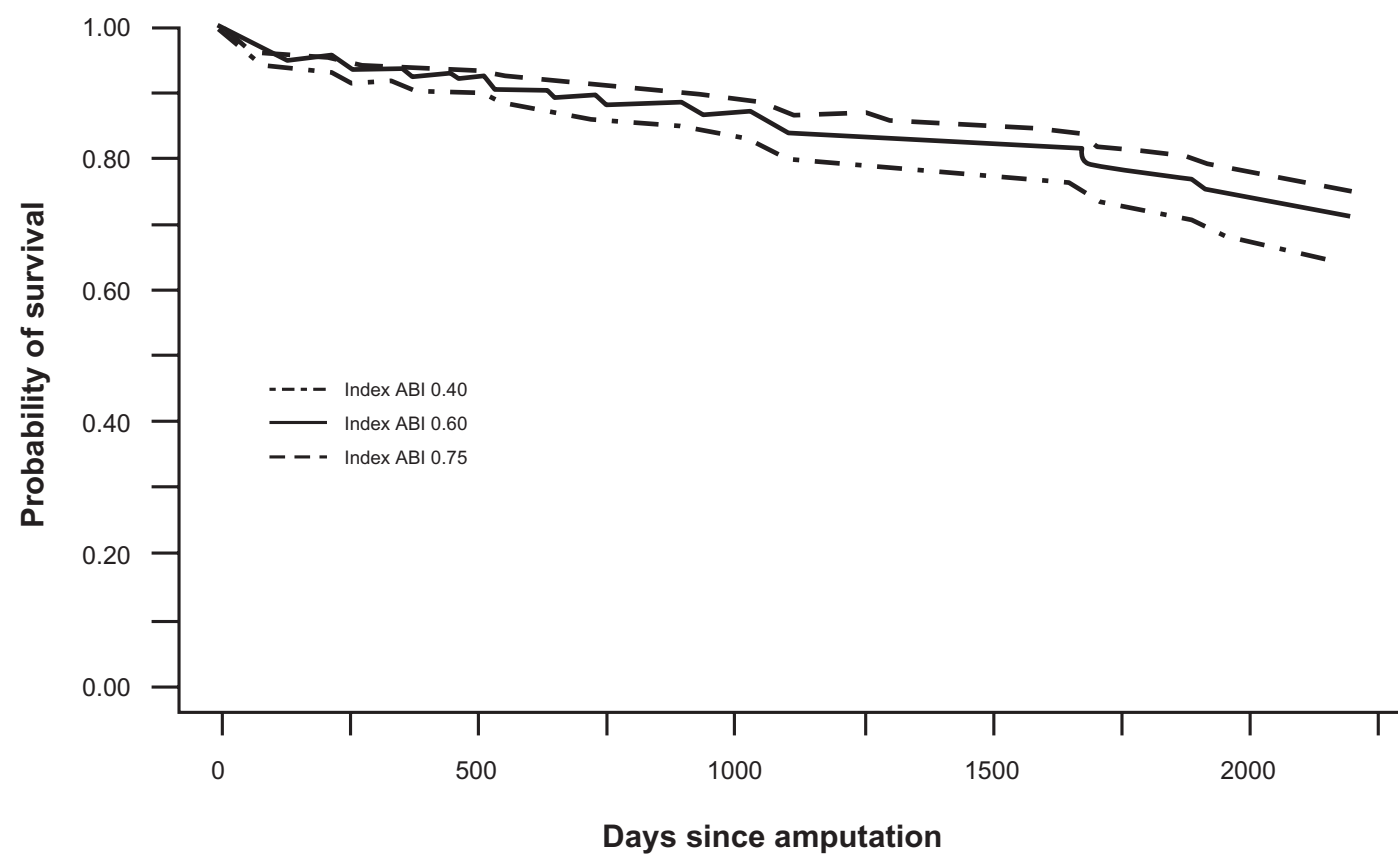

Figure 5 Model-based estimated survival curves for survival after amputation by index ABI. Abbreviation: $\mathrm{ABI}$, ankle-brachial index.

elective surgeries such as lower extremity bypass surgery will also need to increase.

PAD severity is often defined by the ABI with lower levels indicating more severe disease. Prior studies have documented the association of the ABI with cardiovascular morbidity and mortality. ${ }^{721-23}$ The lower the ABI, the more severe the PAD, indicating more advanced atherosclerosis within the lower limb arterial beds. This more severe form of the disease is also a known marker for atherosclerosis in other vascular beds, most notably the coronary arteries, but also the carotid and intracerebral arteries. The more severe the PAD, the more limited the lower limb function with patients having

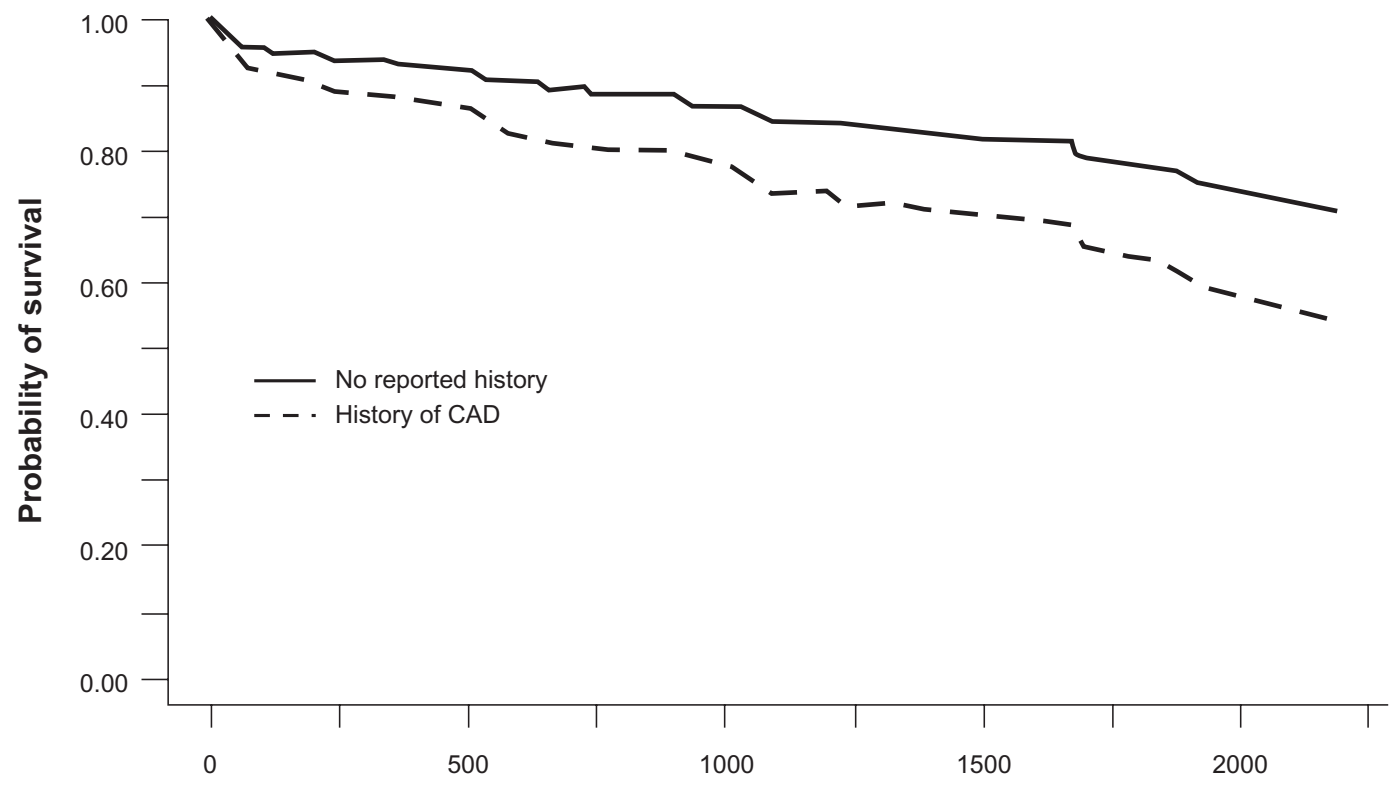

Days since amputation

Figure 6 Model-based estimated survival curves for survival after amputation by CAD history. Abbreviation: CAD, coronary artery disease. 


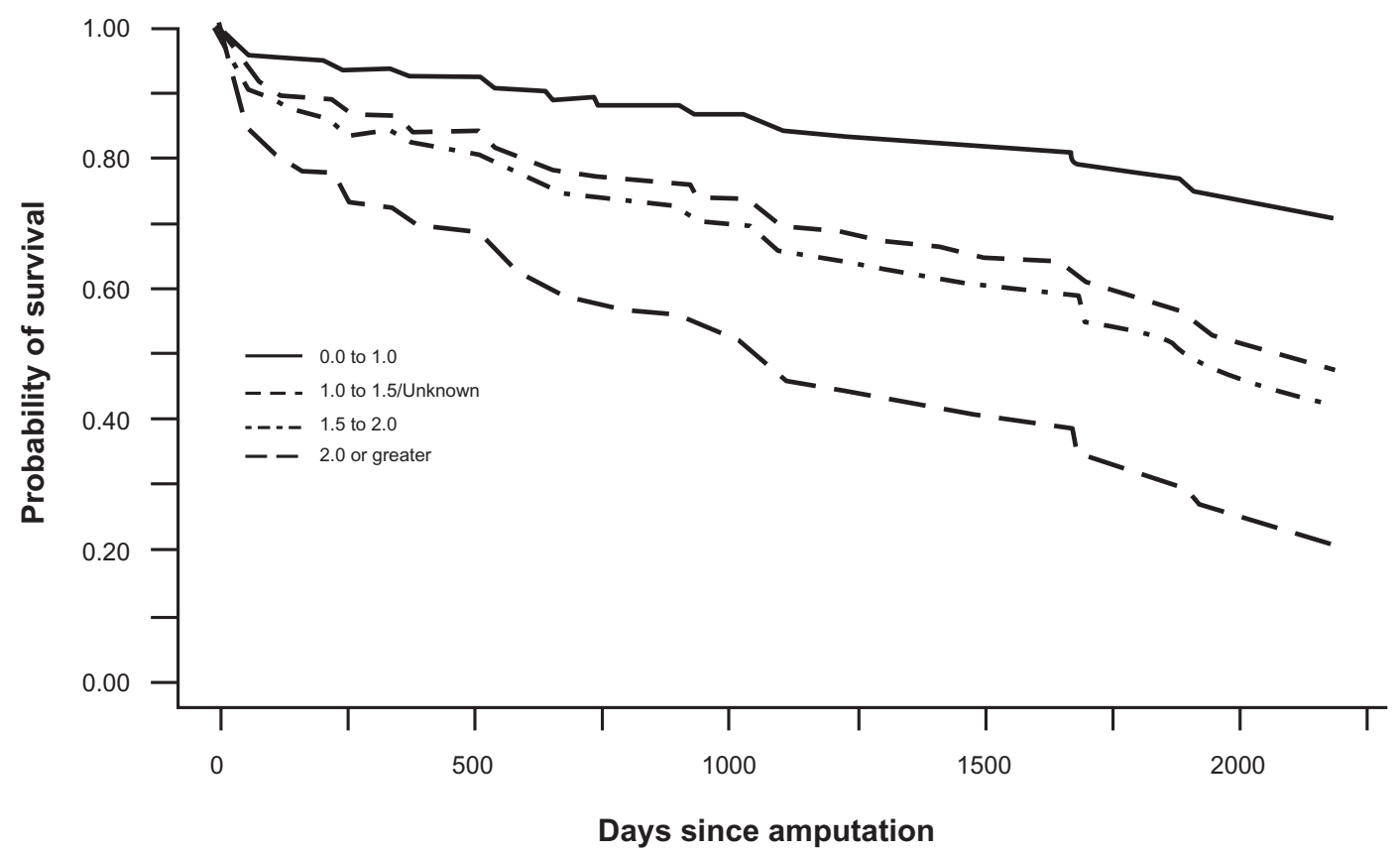

Figure 7 Model-based estimated survival curves for survival after amputation by serum creatinine.

leg discomfort with limited ambulation or even at rest. ${ }^{24,25}$ Thus, the call for more aggressive surgical interventions in patients with severe PAD is not surprising. In our study, PAD severity was a marker for mortality following lower limb revascularization. This finding highlights the severity of co-existing atherosclerosis in vascular beds outside of the lower limbs and the necessity for continued aggressive atherosclerotic risk management.

Renal insufficiency is a known risk factor for coronary heart disease and stroke. ${ }^{26,27}$ Persons with renal insufficiency are also more likely to have coexisting and more severe PAD. In our prior work, renal insufficiency was shown to be a signficant risk factor for lower limb bypass surgery and lower limb amputation. ${ }^{10,28}$ For the present study, we highlight the association of renal insufficiency with increased mortality. Of note, even a serum creatinine in the range of normal to mild elevation was associated with an increased risk for mortality following lower extremity amputation. Reasons for our findings are not yet defined, but are likely multifactorial. Prior work has shown that even mild renal insufficiency is associated with increased cardiovascular mortality. ${ }^{29-31}$ These findings remain after adjustment for coexistent risk factors. We know that metabolic abnormalities are associated with impaired renal function and increase the risk for myocardial dysfunction and damage. Within our cohort, at least one-third of veterans with PAD had some degree of renal impairment while at least $50 \%$ of persons undergoing either lower limb bypass or lower limb amputation had some degree of renal impairment. The presence of renal impairment is likely an additional indicator of PAD severity and introduces additional unmeasured confounders within our study.

While not statistically significant, both current and nonsmokers had a lower risk for mortality following surgery as compared to persons whose smoking status was not assessed. Persons whose smoking status was not assessed were either not abstaining from smoking and possibly had a very high pack-year smoking history or had some other unmeasured variable that increased their risk for mortality. This lack of assessment reflects variability in physician documentation of smoking status and questions of reliability with patient selfreports of smoking status. Clearly, an assessment of smoking status prior to surgery for PAD can lead to better optimization of this risk variable and improve long-term survival.

Our results are from a retrospective cohort study involving data abstraction from electronic and paper medical records. The data capture risk factor prevalence, disease severity, medication use, laboratory values, and physician documentation of recommended care. Many of these variables are process-of-care measures. Each process measure captures a clinician's efforts to treat a modifiable risk factor and a patient's adherence to recommended therapy as well as an actual change in the risk factor of interest, and unmeasured confounding factors. Our smoking control variable is an excellent example of a process-of-care measure. The actual 
variable reflects not only the frequency of physician documentation for this risk factor, but patient self-report of smoking cessation, and actual smoking cessation.

Admittedly, our study has some limitations. First, regarding each process-of-care measure, we cannot tease apart which of the actual processes are presented in our findings. Second, given our retrospective cohort design, we are unable to evaluate other potentially important co-variables such as care obtained outside of the VA, medication adherence, and family support. Third, documentation bias is also a limitation given our use of paper charts and electronic medical records. In spite of the above limitations, our findings highlight that certain risk factors are associated with increased mortality following limb surgery for PAD.

\section{Conclusion}

In conclusion, PAD is a debilitating disease with an associated increased for sugical limb events and death. Our data provide information which surgeons can use to inform patients regarding risk for mortality following lower limb surgery for PAD.

\section{Acknowledgments/disclosures}

This work was supported by an Investigator-Initiated Grant (\#01-180-1; Principal Investigator, Dr Tracie C Collins) from Department of Veterans Affairs, Health Services Research and Development along with resources from the Houston Center for Quality of Care and Utilization Studies, Michael E DeBakey Veterans Affairs Medical Center. Dr Collins was a recipient of the Department of Veterans Affairs Advanced Clinical Research Career Development Award at the time the data for this work was collected. The authors report no conflicts of interest with this work.

\section{References}

1. Hiatt W. Medical treatment of peripheral arterial disease and claudication. NEJM. 2001;344:1608-1621.

2. Stewart KJ, Hiatt WR, Regensteiner JG, Hirsch AT. Exercise training for claudication. 347 ed. 2002. p. 1941-1951.

3. Hirsch AT, Haskal ZJ, Hertzer NR, et al. ACC/AHA 2005 Practice guidelines for the management of patients with peripheral arterial disease (lower extremity, renal, mesenteric, and abdominal aortic): a collaborative report from the American Association for Vascular Surgery/Society for Vascular Surgery, Society for Cardiovascular Angiography and Interventions, Society for Vascular Medicine and Biology, Society of Interventional Radiology, and the ACC/AHA Task Force on Practice Guidelines (Writing Committee to Develop Guidelines for the Management of Patients With Peripheral Arterial Disease): endorsed by the American Association of Cardiovascular and Pulmonary Rehabilitation; National Heart, Lung, and Blood Institute; Society for Vascular Nursing; TransAtlantic Inter-Society Consensus; and Vascular Disease Foundation. Circulation. 2006 Mar 21;113(11): e463-e654.
4. Pell JP, Donnan PT, Fowkes FG, Ruckley CV. Quality of life following lower limb amputation for peripheral arterial disease. Eur J Vasc Surg. 1993;7(4):448-451.

5. Norgren L. [New international consensus document on peripheral arterial disease. TASC II for improved care]. Lakartidningen. 2007;104(19):1474-1475.

6. Morise AP, McDowell DE, Savrin RA, et al. The prediction of cardiac risk in patients undergoing vascular surgery. Am J Med Sci. 1987;293(3):150-158.

7. Fowkes F, Housley E, Cawood E, Macintyre C, Ruckley C, Prescott R. Edinburgh Artery Study: prevalence of asymptomatic and symptomatic peripheral arterial disease in the general population. Int J Epidemiol. 1991;20(2):384-392.

8. Fowkes F, Housley E, Riemersma R, et al. Smoking, lipids, glucose intolerance, and blood pressure as risk factors for peripheral atherosclerosis compared with ischemic heart disease in the Edinburgh Artery Study. Am J Epidemiol. 1992;135(4):331-340.

9. Hirsch A, Criqui M, Treat-Jacobson D, et al. Peripheral arterial disease detection, awareness, and treatment in primary care. JAMA. 2001;286:1317-1324.

10. Collins TC, Beyth RJ, Nelson DB, et al. Process of care and outcomes in patients with peripheral arterial disease. J Gen Intern Med. 2007; 22(7):942-948.

11. The Sixth Report of the Joint National Committee on Prevention, Detection, Evaluation, and Treatment of High Blood Pressure. National Institutes of Health: National Heart, Lung, and Blood Institute, National High Blood Pressure Education Program. 1997. Report No:98-4080.

12. Executive summary of the third report of the National Cholesterol Education Program (NCEP) expert panel on detection, evaluation, and treatment of high blood cholesterol in adults (adult treatment panel III). JAMA. 2001;285(19):2486-2497.

13. American Diabetes Association Consensus Statement: Peripheral arterial disease in people with diabetes. Diabetes Care. 2003;26(12): 3333-3341.

14. McAlister AL, Rabius V, Geiger A, Glynn TJ, Huang P, Todd R. Telephone assistance for smoking cessation: one year cost effectiveness estimations. Tob Control. 2004;13(1):85-86.

15. Stevenson RB. American Cancer Society and smoking cessation. J Am Dent Assoc. 1989;118(3):274.

16. Austin PC, Tu JV. Automated variable selection methods for logistic regression produced unstable models for predicting acute myocardial infarction mortality. J Clin Epidemiol. 2004;57(11):1138-1146.

17. Greenland S. Modeling and variable selection in epidemiological analysis. Am J Public Health. 1989;79(3):340-349.

18. Mickey RM, Greenland S. The impact of confounder selection criteria on effect estimation. Am J Epidemiol. 1989;129(1):125-137.

19. Collins T, Beyth R. Process of care and outcomes in peripheral arterial disease. 325 ed. 2003;125-134.

20. Kannel W. Risk factors for atherosclerotic cardiovascular outcomes in different arterial territories. J Cardiovasc Risk. 1994;1(4):333-339.

21. McKenna M, Wolfson S, Kuller L. The ratio of ankle and arm arterial pressure as an independent predictor of mortality. Atherosclerosis. 1991;87:119-128.

22. Newman A, Siscovick D, Manolio T, et al. Ankle-arm index as a marker of atherosclerosis in the Cardiovascular Health Study. Cardiovascular Heart Study (CHS) Collaborative Research Group. Circulation. 1993;88(3):837-845.

23. Newman A, Tyrrell K, Kuller L. Mortality over four years in SHEP participants with a low ankle-arm index. J Am Geriatr Soc. 1997; 45(12):1472-1478.

24. McDermott M, Mehta S, Liu K, et al. Leg symptoms, the ankle-brachial index, and walking ability in patients with peripheral arterial disease. J Gen Intern Med. 1999;14(3):173-181.

25. McDermott M, Greenland P, Liu K, et al. The ankle-brachial index is associated with leg function and physical activity: the walking and leg circulation study. Ann Intern Med. 2002;136:873-883. 
26. Gambaro G, Verlato F, Budakovic A, et al. Renal impairment in chronic cigarette smokers. J Am Soc Nephrol. 1998;9(4):562-567.

27. Fried LF, Shlipak MG, Crump C, et al. Renal insufficiency as a predictor of cardiovascular outcomes and mortality in elderly individuals. $\mathrm{J} \mathrm{Am}$ Coll Cardiol. 2003;41(8):1364-1372.

28. Collins T, Johnson M, Henderson W, Khuri S, Daley J. Lower extremity non-traumatic amputation among veterans with peripheral arterial disease: is race an independent factor. Med Care. 2002;40 [supplement]: I-106-I-116.
29. Stam F, Van GC, Becker A, et al. Endothelial dysfunction contributes to renal function-associated cardiovascular mortality in a population with mild renal insufficiency: the Hoorn study. J Am Soc Nephrol. 2006;17(2):537-545.

30. Henry RM, Kostense PJ, Bos G, et al. Mild renal insufficiency is associated with increased cardiovascular mortality: The Hoorn Study. Kidney Int. 2002;62(4):1402-1407.

31. Culleton BF, Larson MG, Wilson PW, Evans JC, Parfrey PS, Levy D. Cardiovascular disease and mortality in a community-based cohort with mild renal insufficiency. Kidney Int. 1999;56(6):2214-2219.

\section{Publish your work in this journal}

Vascular Health and Risk Management is an international, peerreviewed journal of therapeutics and risk management, focusing on concise rapid reporting of clinical studies on the processes involved in the maintenance of vascular health; the monitoring, prevention and treatment of vascular disease and its sequelae; and the involvement of metabolic disorders, particularly diabetes. This journal is indexed on PubMed Central and MedLine. The manuscript management system is completely online and includes a very quick and fair peer-review system, which is all easy to use. Visit http://www.dovepress.com/ testimonials.php to read real quotes from published authors.

Submit your manuscript here: http://www.dovepress.com/vascular-health-and-risk-management-journal 